Research Article

\title{
Benefit of Relaxation-Type Damping on the Performance of a Six-Degree-of-Freedom Microvibration Isolation Device for Control Moment Gyroscope
}

\author{
Xingtian Liu $\mathbb{D}^{\mathbb{D}}$, Changbao Shao $\mathbb{D}$, Liping Zhou, and Xiangsen Kong \\ Laboratory of Space Mechanical and Thermal Integrative Technology, Shanghai Institute of Satellite Engineering, \\ Shanghai 201109, China \\ Correspondence should be addressed to Xingtian Liu; xtliu509@126.com
}

Received 8 October 2020; Revised 14 February 2021; Accepted 11 March 2021; Published 8 April 2021

Academic Editor: Zeqi Lu

Copyright (C) 2021 Xingtian Liu et al. This is an open access article distributed under the Creative Commons Attribution License, which permits unrestricted use, distribution, and reproduction in any medium, provided the original work is properly cited.

In order to provide an ultraquiet environment for spacecraft payload, a six-degree-of-freedom microvibration isolation device for satellite control moment gyro (CMG) is proposed in this paper. The dynamic characteristics of the microvibration isolation device are analyzed theoretically and experimentally. The dynamic equations of the microvibration suppression device are established by using the Newton-Euler method. The dynamic responses are numerically solved and the frequency-domain characteristics of the microvibration isolation device under base excitation are analyzed. The analytical results are first verified numerically, and the two results are in good accordance. The experimental apparatus is built, and the vibration isolation performance is investigated. The acceleration transfer function is measured and the influence of the excitation amplitude on the vibration isolation performance is performed. It is shown that the amplification factor at the vicinity of the resonance frequency is within $10 \mathrm{~dB}$, and the vibration isolation performance is significant at higher frequencies. The vibration attenuation performance at the main frequency of the CMG $(100 \mathrm{~Hz})$ is more than $30 \mathrm{~dB}$. The microvibration suppression device can effectively suppress the microvibration generated by CMG during orbital operation.

\section{Introduction}

Remote sensing or observation of the Earth is one of the most important tasks of satellite. In order to get an ultra-high-resolution image, many disturbances that affect the performance of the sensitive payload need to be controlled on board. The most annoying disturbance that degrades the performance of payload is thermal deflection $[1,2]$ of satellite structure and microvibration ejected by moving parts such as cryocooler [3-5], flywheel $[6,7]$, and control moment gyroscope (CMG) [8]. As a matter of fact, CMGs are widely used in fast maneuver satellites because of their significant angular momentum. Nevertheless, the high-speed rotors in CMGs also make them become one of the largest microvibration sources. In a recently published paper [9], the on-orbit microvibration measurement in a remotesensing satellite shows that flywheel and CMG are the main vibration source.
Therefore, it is urgent to isolate the microvibration generated by flywheel and CMGs in order to provide quiet environment for sensitive payload. Not surprisingly, many microvibration suppression methods including vibration source control and payload isolation [10] are employed to achieve the purpose. For example, the famous Hubble Telescope used the viscous damper developed by Honeywell to isolate flywheel vibration [11]. In Chandra X-ray Observatory [12], the Stewart-type passive vibration isolation device for flywheel is also considered, and the vibration isolation performance of which at the launch phase is also designed and tested. Li et al. [13] conducted research on the microvibration induced by flywheel and the effect on space camera; the experimental results on ground show that the camera can work normally after a vibration isolator is applied to the flywheel. Cobb et al. [14] proposed a passiveactive vibration isolation system to protect the optical 
payload from the unwanted microvibration on board. Spanos et al. [15] at JPL invented a flexible active vibration isolation and pointing system which was successfully applied in James Webb Space Telescope. Zhang et al. [16, 17] proposed a new method to achieve better image quality of optical payload. In their research, both the control strategy and the passive vibration source isolation are implemented. Li et al. [18] also considered a similar system and the difference of which laying on the vibration isolation system is active. Zhang et al. [19] focuse on the design of internal vibration isolation structures for the CMG to reduce the vibration generated by the imbalance of the rotor. A fully passive two-stage isolation system based on viscoelastic materials has been developed in order to minimize the CMG microvibrations by Kawak [20]. This paper proposed a new type of vibration isolation device to isolate the CMG vibration transmitted to satellite. The device not only can attenuate vibrations at the main frequency of the CMG but also can suppress resonance amplification factor to a considerable low level. Some useful design guidelines and concrete engineering based experimental results can enrich the vibration isolation field for CMGs.

This paper is organized as follows. Firstly, the dynamic model of the proposed vibration isolation device based on a relaxation-type damper is deduced using Euler-Newton method. The dynamic equation is then solved numerically. Secondly, the vibration isolation performances for all six degrees of freedom are analyzed and the coupling features in different directions are discussed. Thirdly, the experimental setup is built, and the vibration isolation performance is evaluated in three translational directions. Some valuable conclusions are drawn in the last section.

\section{Dynamic Modeling}

2.1. The Microvibration Isolation Device. The Stewart-type structure is used very popularly to get vibration isolation performance in six degrees of freedom [21]. The schematic of the proposed microvibration isolation device for single CMG in this paper is shown in Figure 1, which is also based on a Stewart-type structure. The platform includes upper platform to connect with the CMG and the lower platform to be installed on the satellite. In between, six relaxation-type isolators are configured in a cubic pattern, which provides proper stiffness and damping to isolate the vibration generated by the CMG transmitting to the satellite. Figure 2 shows a simplified representation of the vibration isolation device in which some useful coordinates are defined. The local coordinates on the upper and lower platforms are $P x_{p} y_{p} z_{p}$ and $B x_{b} y_{b} z_{b}$, respectively. The global inertial coordinate system is Gxyz. The hinges of the upper and lower platforms with the isolator are defined as $P_{i} x_{p i} y_{p i} z_{p i}$ and $B_{i} x_{b i} y_{b i} z_{b i}$. Oxyz is the inertial principal axis coordinate system of the CMG.

The following symbols are used to build the dynamic equation of the system. $\mathbf{t}_{p}$ is the position vector in coordinate $G x y z, \mathbf{p}_{i}$ is the position vector of the hinge where the upper

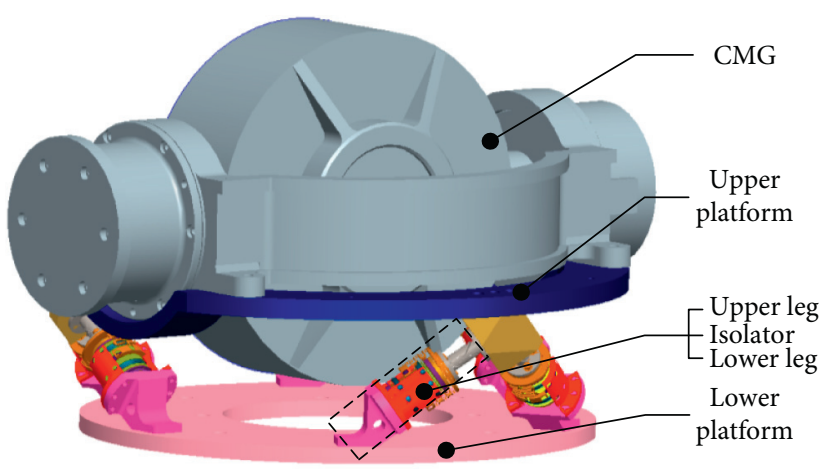

Figure 1: The 3D model of microvibration suppression device.

platform and the isolator connected in coordinate $P x_{p} y_{p} z_{p}$, $\mathbf{b}_{i}$ is the position vector of the hinge where the lower platform and the isolator connected in coordinate $B x_{b} y_{b} z_{b}$, $\mathbf{t}_{i}$ is the length vector of each isolator between the upper and lower platforms in coordinate $G x y z, \mathbf{R}_{0}$ is the position vector of the mass center of the CMG with the upper platform in coordinate $P x_{p} y_{p} z_{p}, \omega_{p}$ and $\alpha_{p}$ are the angular velocity and angular acceleration of the upper platform, $\omega_{b}$ and $\alpha_{b}$ are the angular velocity and angular acceleration of the lower platform, $\mathbf{I}_{p}$ is the inertial matrix of the CMG with upper platform in coordinate $G x y z$, and $\mathbf{I}_{u 0 i}$ and $\mathbf{I}_{d 0 i}$ are the inertial matrix of the upper and lower legs in coordinates $P x_{p} y_{p} z_{p}$ and $B x_{b} y_{b} z_{b} \cdot \mathbf{r}_{u 0 i}$ and $\mathbf{r}_{d 0 i}$ are the position vectors of the upper and lower legs in coordinates $P x_{p} y_{p} z_{p}$ and $B x_{b} y_{b} z_{b}$.

2.2. Modeling of the Strut and Upper Platform. Different from many researchers who consider the single strut as a Kevin model or an active actuator, the strut used in this paper is a relaxation-type damper. The strut model and the load applied to the strut are shown in Figure 3.

As this vibration isolation device is a Stewart-type platform, the strut is symmetric along the center axis of the upper and lower platforms. The position vector $\mathbf{l}_{i}$ of the $i^{\text {th }}$ strut in coordinate $\{G\}$ can be expressed as

$$
\mathbf{l}_{i}=\mathbf{P}_{i}-\mathbf{B}_{i}=\mathbf{t}_{p i}+\mathbf{t}_{p}-\mathbf{t}_{b i}-\mathbf{t}_{b}
$$

where $\quad \mathbf{t}_{p i}=\mathbf{R}_{p}^{g} \mathbf{p}_{i}, \quad \mathbf{t}_{b i}=\mathbf{R}_{b}^{g} \mathbf{b}_{i}, \quad \mathbf{t}_{p}=\left[\begin{array}{lll}0 & 0 & h\end{array}\right]^{T}, \quad$ and $\mathbf{t}_{b}=\left[\begin{array}{lll}0 & 0 & 0\end{array}\right]^{T}$.

The unit vector for the $i^{\text {th }}$ strut is given as

$$
\boldsymbol{\tau}_{i}=\frac{\mathbf{l}_{i}}{\left|\mathbf{l}_{i}\right|} .
$$

The translational velocity and acceleration along the strut for the $i^{\text {th }}$ strut are

$$
\begin{aligned}
& \mathbf{l}_{i}^{\prime}=\mathbf{t}_{p}^{\prime}+\boldsymbol{\omega}_{p} \times \mathbf{t}_{p i}=\mathbf{v}_{p i}, \\
& \mathbf{l}_{i}^{\prime \prime}=\mathbf{t}_{p}^{\prime \prime}+\boldsymbol{a}_{p} \times \mathbf{t}_{p i}+\boldsymbol{\omega}_{p} \times\left(\boldsymbol{\omega}_{p} \times \mathbf{t}_{p i}\right)=\mathbf{a}_{p_{i}}=\mathbf{a}_{p_{i}}+\mathbf{u}_{1 i},
\end{aligned}
$$

where $\mathbf{u}_{1 i}=\omega_{p} \times\left(\omega_{p} \times \mathbf{t}_{p i}\right)$. 


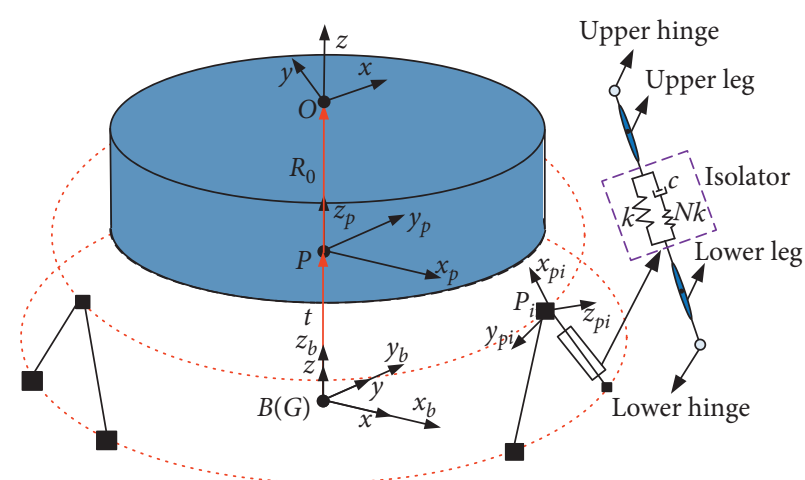

(a)

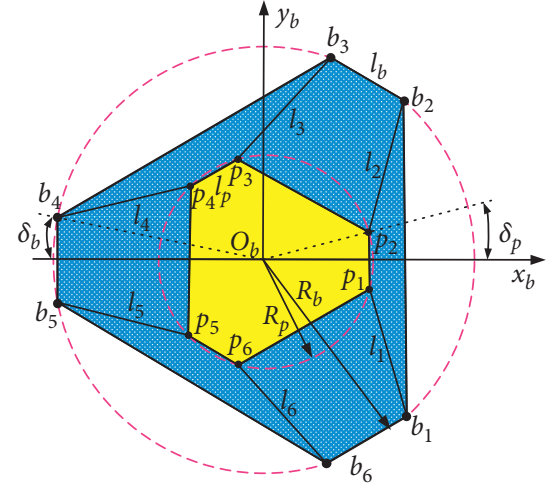

(b)

FIgURE 2: Schematic of the microvibration suppression device. (a) Simplified model. (b) Top view of the model.

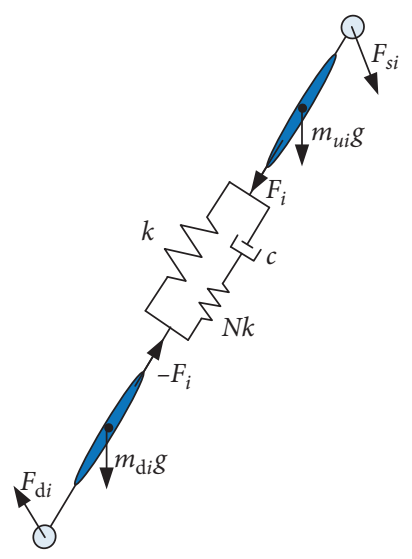

FIgURE 3: Force analysis diagram of the $i^{\text {th }}$ leg.

The acceleration of the mass center of the upper and lower legs can be expressed as

$$
\begin{aligned}
& \mathbf{a}_{u i}=\left(\mathrm{E}_{3}+\frac{1}{l_{i}}\left(\boldsymbol{\tau}_{i}^{T} \mathbf{r}_{u i}-\boldsymbol{\tau}_{i} \mathbf{r}_{u i}^{T}\right)\right) \mathbf{a}_{p i}+\mathbf{u}_{4 i}, \\
& \mathbf{a}_{d i}=\frac{1}{l_{i}}\left(\boldsymbol{\tau}_{i}^{T} \mathbf{r}_{d i}-\boldsymbol{\tau}_{i} \mathbf{r}_{d i}^{T}\right) \mathbf{a}_{p i}+\mathbf{u}_{5 i},
\end{aligned}
$$

where $\mathbf{u}_{4 i}=\omega_{p} \times\left(\omega_{p} \times \mathbf{t}_{p i}\right)+\omega_{i} \times\left(\omega_{i} \times \mathbf{r}_{u i}\right)+\mathbf{u}_{3 i} \times \mathbf{r}_{u i}$ and $\mathbf{u}_{5 i}=\omega_{i} \times\left(\omega_{i} \times \mathbf{r}_{d i}\right)+\mathbf{u}_{3 i} \times \mathbf{r}_{d i}$.

Equations of moment of momentum for the upper and lower legs are given as

$$
\begin{gathered}
\left\{\begin{array}{l}
m_{u i} \mathbf{g}+\mathbf{F}_{s i}+\mathbf{F}_{i}=m_{u i} \mathbf{a}_{u i}, \\
m_{d i} \mathbf{g}+\mathbf{F}_{d i}-\mathbf{F}_{i}=m_{d i} \mathbf{a}_{d i},
\end{array}\right. \\
\left\{\begin{array}{l}
-\mathbf{r}_{u i} \times \mathbf{F}_{s i}=I_{u i} \boldsymbol{\alpha}_{i}+\boldsymbol{\omega}_{i} \times I_{u i} \boldsymbol{\omega}_{i}, \\
-\mathbf{r}_{d i} \times \mathbf{F}_{d i}=I_{d i} \boldsymbol{\alpha}_{i}+\boldsymbol{\omega}_{i} \times I_{d i} \boldsymbol{\omega}_{i} .
\end{array}\right.
\end{gathered}
$$

Equation (6) can be simplified to express the force applied to the upper platform from one single strut, which is

$$
\mathbf{F}_{s i}=\mathbf{Q}_{p i}\left(\mathbf{t}_{p}^{\prime \prime}-\widetilde{t}_{p i} \boldsymbol{\alpha}_{p}\right)+\mathbf{Q}_{b i}\left(\mathbf{t}_{b}^{\prime \prime}-\widetilde{t}_{b i} \boldsymbol{\alpha}_{b}\right)+\mathbf{V}_{i}-f_{i} \boldsymbol{\tau}_{i}
$$

As shown in Figure 4, the force applied to the upper platform from one single strut is $-\mathbf{F}_{s i}$. The gravity of the upper platform and the CMG is $m_{0} \mathbf{g}$, and the force and moment disturbance generated by the CMG are assumed as $\mathbf{F}_{\text {mac }}$ and $\mathbf{M}_{\text {mac }}$.

By using Newton-Euler method, the dynamic equation for the upper platform is given as

$$
\left\{\begin{array}{l}
-m_{0} \mathbf{q}_{c}^{\prime \prime}+m_{0} \mathbf{g}+\mathbf{R}_{p}^{g} \mathbf{R}_{o}^{p} \mathbf{F}_{\mathrm{mac}}-\sum_{i=1}^{N} \mathbf{F}_{s i}=0, \\
m_{0} \mathbf{R} \times \mathbf{g}-m_{0} \mathbf{R} \times \mathbf{q}_{c}^{\prime \prime}+\mathbf{R}_{p}^{g} \mathbf{R}_{o}^{p} \mathbf{M}_{\mathrm{mac}}+\mathbf{R}_{p}^{g} \mathbf{R}_{o}^{p} \mathbf{R} \times \mathbf{F}_{\mathrm{mac}}-\mathbf{I}_{p} \boldsymbol{\alpha}_{p}-\boldsymbol{\omega}_{p} \times \mathbf{I}_{p} \boldsymbol{\omega}_{p}-\sum_{i=1}^{N} \mathbf{t}_{p i} \times \mathbf{F}_{s i}=0,
\end{array}\right.
$$

where $\mathbf{q}_{c}^{\prime \prime}$ is the absolute acceleration of the upper platform in coordinate $\{\mathbf{G}\}, \mathbf{R}$ is the position vector of the mass center of the upper platform in coordinate $\{\mathbf{P}\}$, and $\mathbf{I}_{p}$ is the inertia matrix of the upper platform in coordinate $\{\mathbf{G}\}$.
2.3. Response Solution. The force $\mathbf{F}_{i}$ applied to the upper platform from each strut can be expressed as

$$
\mathbf{F}_{i}=-k_{i}\left(l_{i}-l_{i 0}\right) \boldsymbol{\tau}_{i}-c_{i} x_{d i}^{\prime} \boldsymbol{\tau}_{i} c_{i} x_{d}^{\prime}+N_{i} k_{i} x_{d i}=N_{i} k_{i}\left(l_{i}-l_{i 0}\right),
$$




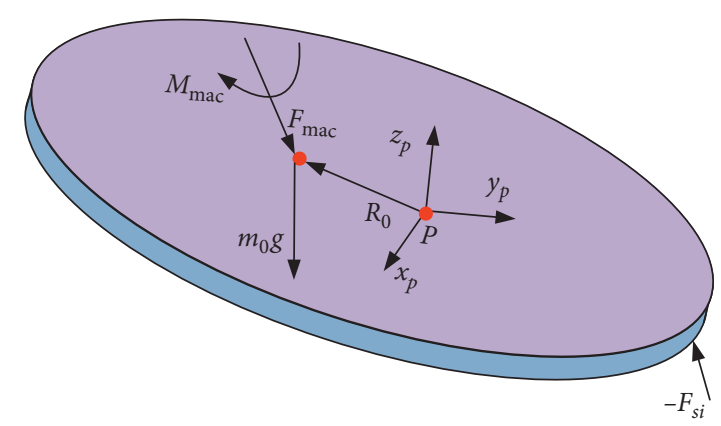

FIgURE 4: Force analysis diagram for the upper platform.

where $x_{d i}$ is the deflection of the joint of the damping and the parallel connected spring in the $i^{\text {th }}$ strut.

Applying Laplace transform to equation (9), one can get the simplified equation

$$
\mathbf{F}_{i}=-\frac{\left(N_{i} k_{i}^{2}+c_{i} k_{i}\left(N_{i}+1\right) s\right)}{\left(N_{i} k_{i}+c_{i} s\right)}\left(\mathbf{J}_{p i} \mathbf{x}_{p}-\mathbf{J}_{b i} \mathbf{x}_{b}\right),
$$

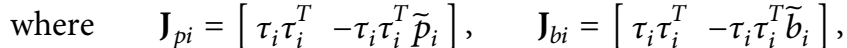
$\mathbf{x}_{p}=\left[\begin{array}{ll}\mathbf{t}_{p}^{T} & \theta_{p}^{T}\end{array}\right]^{T}$, and $\mathbf{x}_{b}=\left[\begin{array}{cc}\mathbf{t}_{b}^{T} & \theta_{b}^{T}\end{array}\right]^{T}$.

Combining equations (7)-(9), the dynamic equation of the vibration isolation device subjected to base excitation can be derived asw

$$
\left(N_{i} k_{i}+c_{i} s\right) \mathbf{M}_{p} \mathbf{x}_{p}^{\prime \prime}+\mathbf{C}_{p} \mathbf{x}_{p}^{\prime}+\mathbf{K}_{p} \mathbf{x}_{p}=\left(N_{i} k_{i}+c_{i} s\right) \mathbf{M}_{b} \mathbf{x}_{b}^{\prime \prime}+\mathbf{C}_{b} \mathbf{x}_{b}^{\prime}+\mathbf{K}_{b} \mathbf{x}_{b}+\left(N_{i} k_{i}+c_{i} s\right) \mathbf{U}
$$

where

$$
\begin{aligned}
& \mathbf{M}_{p}=\left[\begin{array}{cc}
m_{0} \mathbf{E}_{3} & -m_{0} \widetilde{R} \\
m_{0} \widetilde{R} & \mathbf{I}_{p}-m_{0} \widetilde{R} \widetilde{R}
\end{array}\right]+\sum_{i=1}^{N}\left[\begin{array}{cc}
\mathbf{Q}_{p i} & -\mathbf{Q}_{p i} \widetilde{t}_{p i} \\
\widetilde{t}_{p i} \mathbf{Q}_{p i} & -\widetilde{t}_{p i} \mathbf{Q}_{p i} \widetilde{t}_{p i}
\end{array}\right], \\
& \mathbf{C}_{p}=\sum_{i=1}^{N} c_{i} k_{i}\left(N_{i}+1\right)\left[\begin{array}{c}
\mathbf{J}_{p i} \\
\widetilde{t}_{p i} \mathbf{J}_{p i}
\end{array}\right], \quad \mathbf{K}_{p}=N_{i} k_{i}^{2}\left[\begin{array}{c}
\mathbf{J}_{p i} \\
\widetilde{t}_{p i} \mathbf{J}_{p i}
\end{array}\right], \\
& \mathbf{M}_{b}=\sum_{i=1}^{N}\left[\begin{array}{cc}
\mathbf{Q}_{b i} & -\mathbf{Q}_{b i} \widetilde{t}_{b i} \\
\widetilde{t}_{p i} \mathbf{Q}_{b i} & -\widetilde{t}_{p i} \mathbf{Q}_{b i} \widetilde{t}_{b i}
\end{array}\right], \quad \mathbf{K}_{b}=\sum_{i=1}^{N} N_{i} k_{i}^{2}\left[\begin{array}{c}
\mathbf{J}_{b i} \\
\widetilde{t}_{b i} \mathbf{J}_{b i}
\end{array}\right], \\
& \mathbf{C}_{b}=\sum_{i=1}^{N} c_{i} k_{i}\left(N_{i}+1\right)\left[\begin{array}{c}
\mathbf{J}_{b i} \\
\widetilde{t}_{b i} \mathbf{J}_{b i}
\end{array}\right], \\
& \mathbf{U}=\left[\begin{array}{c}
\mathbf{R}_{p} \mathbf{F}_{\text {mac }} \\
\mathbf{R}_{p} \mathbf{M}_{\mathrm{mac}}+\mathbf{R}_{p} \mathbf{R} \times \mathbf{F}_{\mathrm{mac}}
\end{array}\right]-\eta .
\end{aligned}
$$$$
\text { Equation (11) gives the dynamic responses of the upper }
$$$$
\text { platform when the base is excited with harmonic vibration. }
$$

\section{Performance of the Microvibration Isolation Device}

The microvibration isolation device is designed to isolate vibration ejected by CMG and the parameters of which are given in Table 1. The relaxation-type damper has been manufactured beforehand and the parameters of which are tested and recorded in Table 2. Meanwhile, the structure parameters of the microvibration isolation device are summarized in Table 3.

3.1. Natural Frequency Analysis. First, the natural frequencies of the microvibration isolation in six directions are analyzed. By simplifying equation (11) and neglecting damping, one can get the free vibration equation of the isolation device without damping as

$$
N_{i} k_{i} \mathbf{M}_{p} \mathbf{x}_{p}^{\prime \prime}+\mathbf{K}_{p} \mathbf{x}_{p}=0
$$

In equation (12), $\mathbf{K}_{p} x$ is the equivalent stiffness matrix and $N_{i} k_{i} \mathbf{M}_{p}$ is the equivalent mass matrix. The first six-order natural frequencies of the vibration isolation system can be obtained by solving equation (12). The modal analysis is also conducted by using finite element software and the model of which is shown in Figure 5. The results of the natural frequency obtained from the finite element software are compared with the theoretical results solved from equation (12). The comparison results are given in Table 4 and the two results are in good accordance. Moreover, in order to understand the natural characteristics of this system thoroughly, the corresponding mode shapes are also summarized in Figure 6.

From the results of natural frequency and the mode shape, one can conclude that the third-order mode and the fifth-order mode are the $Z$-direction translational and $Z$-axis twist pure mode, respectively. The other four modes are coupled mode in $X$-direction and $Y$-direction.

3.2. Relaxation-Type Damper Performance. As mentioned above, the flexible strut used here is a relaxation-type damper. The advantage of this kind of damper over a Kevin model damper is that it can control the resonance amplification without losing capacity of attenuating higher-frequency vibration if the parameters of the damper are chosen carefully. To clarify this point more clearly, the transmissibility of the relaxation-type damper used in this paper is plotted in Figure 7. For the sake of comparison convenience, the transmissibility curve of a system with a Kevin model is also plotted in the same figure. One can see that although the two dampers exhibit the 
TABle 1: Parameters of the CMG.

\begin{tabular}{lc}
\hline Parameters & Data \\
\hline$m$ & $25.22 \mathrm{Kg}$ \\
$I_{x}$ & $0.4469 \mathrm{Kg} \cdot \mathrm{m}^{2}$ \\
$I_{y}$ & $0.1956 \mathrm{Kg} \cdot \mathrm{m}^{2}$ \\
$I_{z}$ & $0.6074 \mathrm{Kg} \cdot \mathrm{m}^{2}$ \\
\hline
\end{tabular}

TABle 2: Parameters of the relaxation-type damper.

\begin{tabular}{lc}
\hline Parameters & Data \\
\hline$k$ & $19.2 \mathrm{~N} / \mathrm{mm}$ \\
$c$ & $1300 \mathrm{~N} \cdot \mathrm{s} / \mathrm{m}$ \\
$N$ & 4 \\
\hline
\end{tabular}

TABle 3: Parameters of the vibration suppression device.

\begin{tabular}{lc}
\hline Parameters names & $\begin{array}{c}\text { Data/ } \\
\mathrm{mm}\end{array}$ \\
\hline $\begin{array}{l}\text { Circumcircle of hinges on upper platform radius } R_{p} \\
\text { Circumcircle of hinges on the lower platform radius } R_{b}\end{array}$ & $\begin{array}{c}187 \\
\text { Shorter distance of hinges on upper platform } l_{p} \text { (see }\end{array}$ \\
$\begin{array}{l}\text { Figure 2(b)) } \\
\text { Shorter distance of hinges on upper platform } l_{b} \text { (see }\end{array}$ & 149 \\
$\begin{array}{l}\text { Figure 2(b)) } \\
\text { Height of the device } h\end{array}$ & 76 \\
Mass center of the CMG to the upper platform $H$ & 40 \\
\hline
\end{tabular}

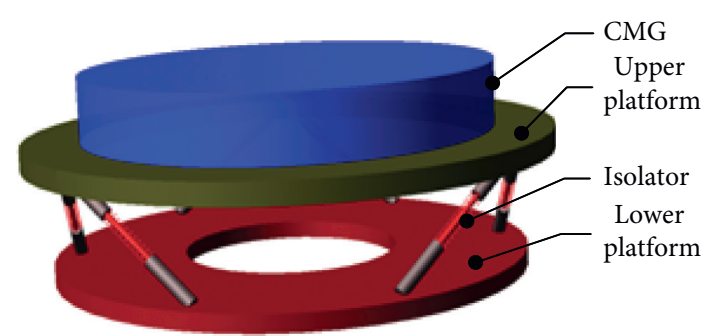

Figure 5: Finite element model of the microvibration suppression device.

TABLE 4: Comparison of nature frequencies solved by analytical and numerical methods.

\begin{tabular}{lccc}
\hline Modal & Theoretical result & Numerical result & Relative error \\
\hline 1 & 8.1550 & 7.7400 & 5.08 \\
2 & 8.8524 & 8.5763 & 3.11 \\
3 & 13.3529 & 12.0770 & 9.55 \\
4 & 16.6622 & 17.0831 & -2.52 \\
5 & 18.2286 & 18.6618 & -2.37 \\
6 & 22.0580 & 21.8344 & -1.01 \\
\hline
\end{tabular}

same resonance amplification factor, the vibration attenuation rate at the main frequency of CMG $(100 \mathrm{~Hz})$ has more than $10 \mathrm{~dB}$ differences, which is the advantage of the relaxationtype damping.

3.3. Coupling Analysis. The main reason that induced the coupling in different directions for the vibration isolation device considered here is that the mass center is out of the plane of the upper platform. Without loss of generality, the parameters $h=0 \mathrm{~mm}$ and $h=40 \mathrm{~mm}$ are chosen to illustrate the coupling characteristic of the device. The transmissibility of the two cases is plotted in Figures 8 and 9 for base excitation.

By observing the two figures, one can conclude that if the mass center of the CMG is in the plane of upper platform, there is no coupling for the device. When the mass center of the CMG is higher than the plane of upper platform, the $X$-direction translation, $Y$-direction rotation, $Y$-direction translation, and $X$-direction rotation are coupled. Two peaks in the transmissibility are observed. However, the movements in $Z$-direction translation and rotation are independent of the other direction in spite of the changing of the mass center of the CMG. This conclusion is very important when designing such kind of device to acquire better vibration isolation performance in certain direction.

3.4. Vibration Isolation Performance in Translational and Rotational Direction. To evaluate the vibration isolation performance of the device, equation (13) is numerically solved by the Runge-Kutta method in MATLAB software. The base is applied with an excitation amplitude of $30 \mathrm{mg}$ according to satellite experiment and the frequency range is from 2 to $40 \mathrm{~Hz}$. The absolute transmissibility is used to represent the vibration performance, which is defined as the ratio of acceleration of upper and lower platforms. The translational transmissibility and rotational transmissibility are plotted in Figures 10(a) and 10(b). Meanwhile, the resonance amplification factor and the vibration attenuation rate at the main frequency of the CMG are summarized in Table 5.

The transmissibilities in $X$-direction and $Y$-direction translation are basically the same because of the symmetrical feature of the device. In all six directions, the minimum natural frequency is $6.84 \mathrm{~Hz}$ ( $X$-direction translational) and the maximum natural frequency is $28.65 \mathrm{~Hz}$ ( $Y$-direction rotational). The vibration attenuation rate at $100 \mathrm{~Hz}$ is beyond $30 \mathrm{~dB}$, which means that the vibration isolation efficiency is more than 94\%. Moreover, the resonant amplification factor is below 3 times.

\section{The Experiment}

4.1. The Scheme. The experimental scheme is shown in Figure 11. It includes a microvibration excitation table, the vibration isolation device, accelerometers, LMS data acquisition system, and suspension system.

To cancel the gravity, a soft suspension system is built in Figure 12. The vibration table which simulates the excitation of sweep sine frequency applies the excitation to lower platform; both the accelerations on the vibration table and on the CMG are measured in order to calculate the absolute transmissibility. The translational transmissibilities in $X$ direction, $Y$-direction, and $Z$-direction are tested. 


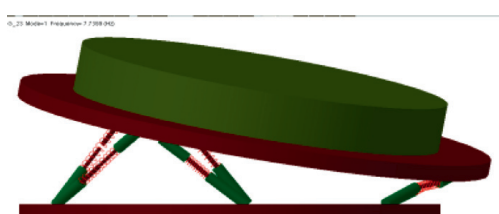

(a)

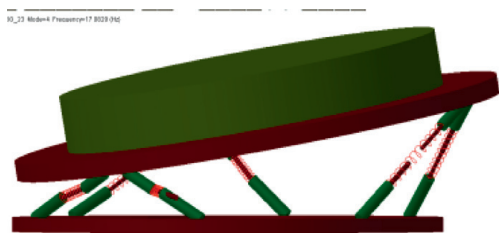

(d)

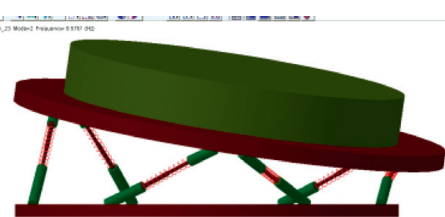

(b)

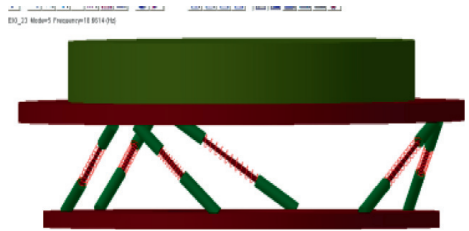

(e)

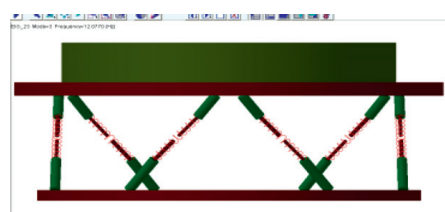

(c)

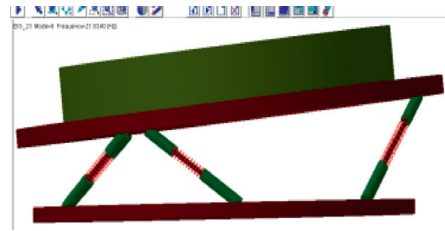

(f)

Figure 6: The first six modes of the microvibration suppression device. (a) First-order mode. (b) Second-order mode. (c) Third-order mode. (d) Fourth-order mode. (e) Fifth-order mode. (f) Sixth-order mode.

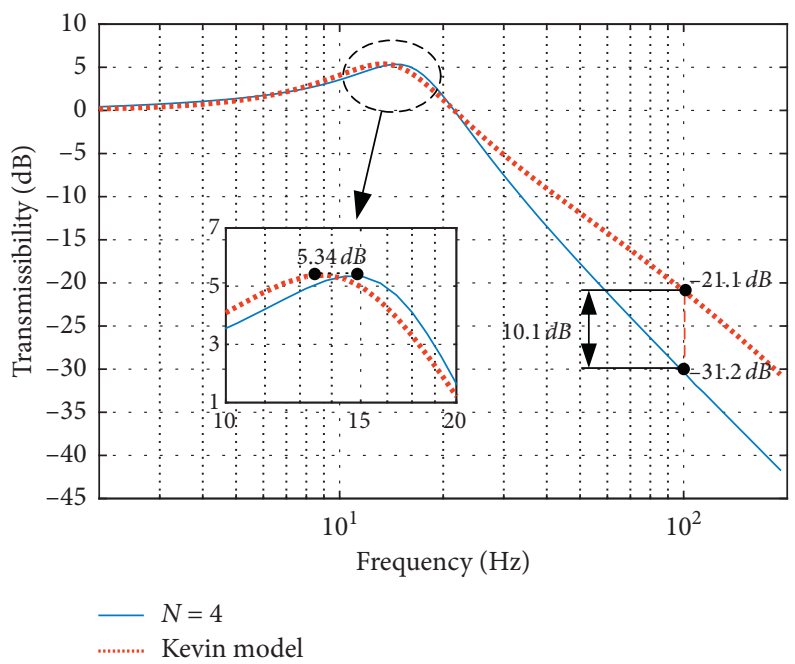

Figure 7: Comparison of the acceleration transmissibility of the relaxation-type damper for $N=4$ and a Kevin model.

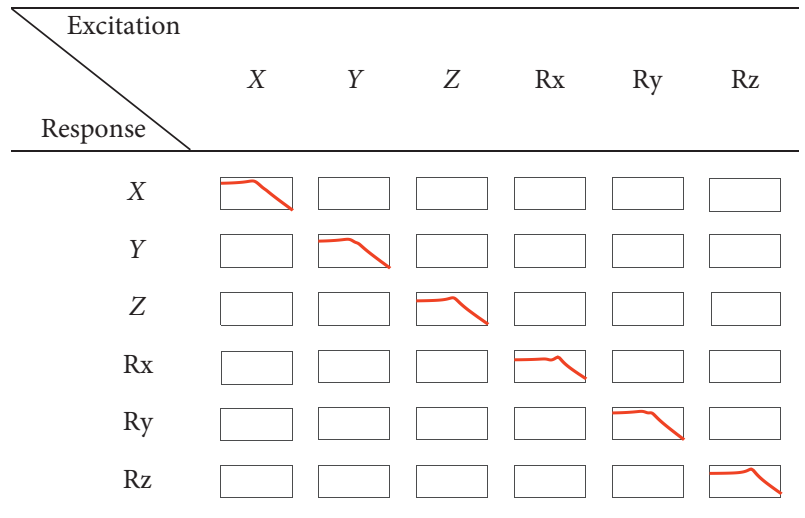

Figure 8: Response of the CMG when $h=0 \mathrm{~mm}$.

4.2. The Soft Suspension System. Four elastic strips are used to suspend the CMG. First, the deflection length and the stiffness of the strip are tested. The force-deflection test

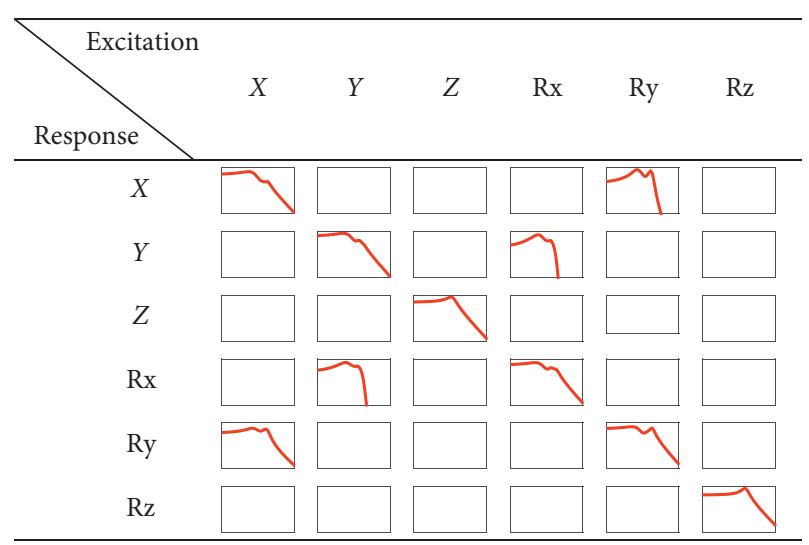

Figure 9: Response of the CMG when $h=40 \mathrm{~mm}$.

apparatus is shown in Figure 13, and the force-deflection curve is fitted by the LMS method, which is given in Figure 14. In the figure, one can calculate that the stiffness of the 


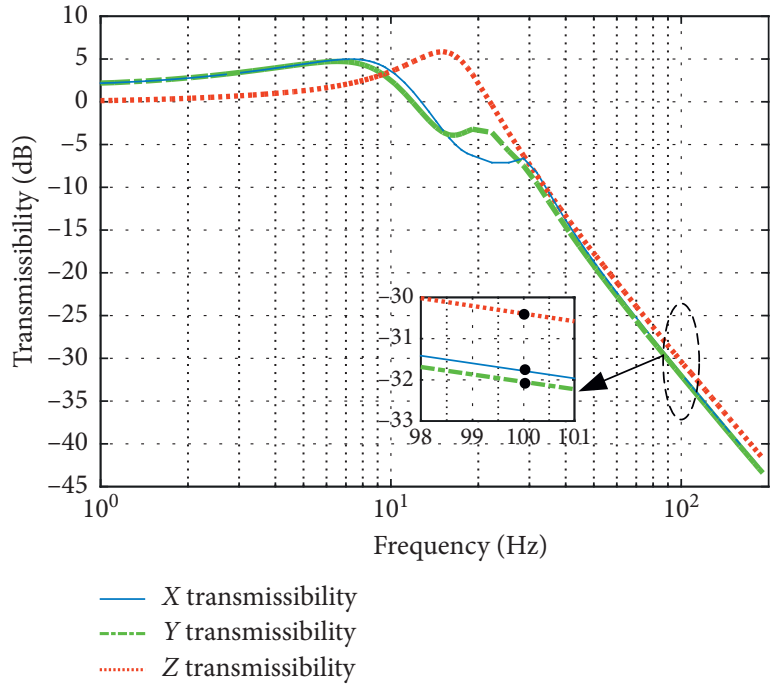

(a)

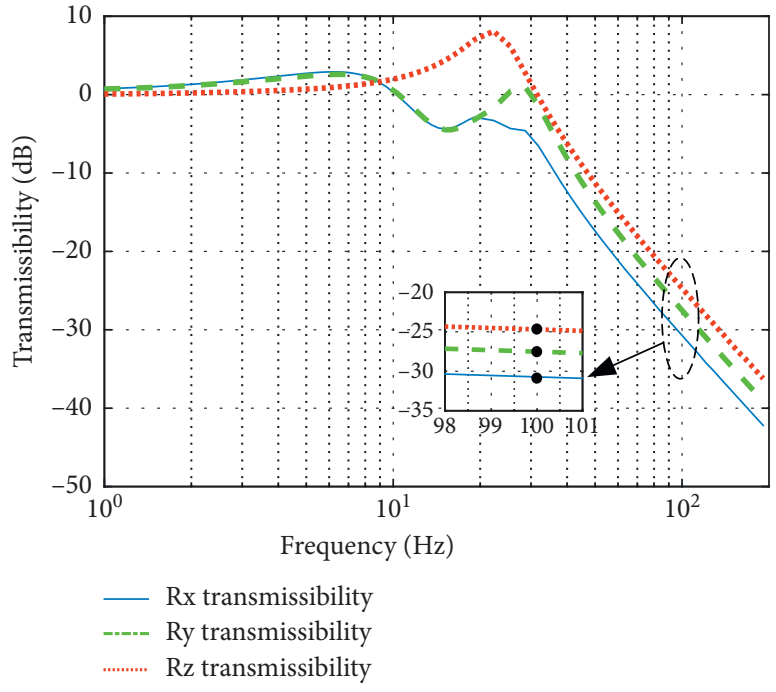

(b)

Figure 10: Transmittance curve of Stewart vibration isolation system in six directions. (a) Transmissibility in the translational direction. (b) Transmissibility in the rotational direction.

TABLE 5: Vibration isolation performance of microvibration suppression device in six directions.

\begin{tabular}{lcccccc}
\hline Direction & $X$ & $Y$ & $Z$ & $\mathrm{Rx}$ & $\mathrm{Ry}$ & $\mathrm{Rz}$ \\
\hline $100 \mathrm{~Hz}$ attenuation rate (\%) & 97 & $98 \%$ & $96 \%$ & $97 \%$ & $96 \%$ & $94 \%$ \\
Resonance amplification factor & 1.75 & 1.72 & 1.95 & 1.39 & 1.34 & 2.53 \\
\hline
\end{tabular}

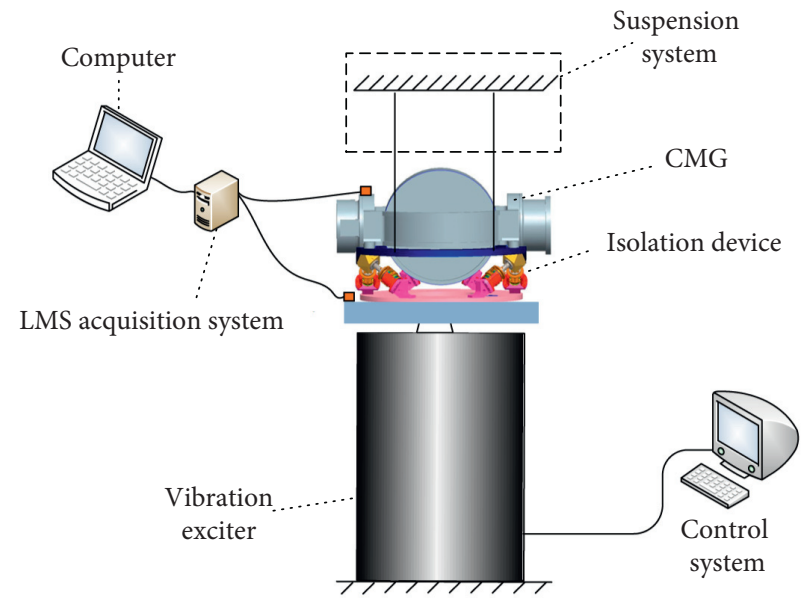

Figure 11: Experimental test system.

strip is about $0.245 \mathrm{~N} / \mathrm{mm}$, which yields a suspension frequency of about $0.3 \mathrm{~Hz}$. The natural frequency of the suspension system is far lower than the first natural frequency of the vibration isolation system, which means that the suspension system can affect the experimental result a little. Moreover, in the vertical direction, the suspension length is more than 3 meters; therefore, the horizontal suspension frequency is also low enough.
4.3. Time-Domain Results. The input and output accelerations measured in the three directions are shown in Figures 15 to 17 . The input signal of the vibration table is a $30 \mathrm{mg}$ amplitude and $2-200 \mathrm{~Hz}$ sine sweep vibration. In the time domain in Figures 15 to 17, one can see clearly the amplification and attenuation of the vibration isolation device. In the $Z$-direction translational excitation, only one peak is observed, which means 


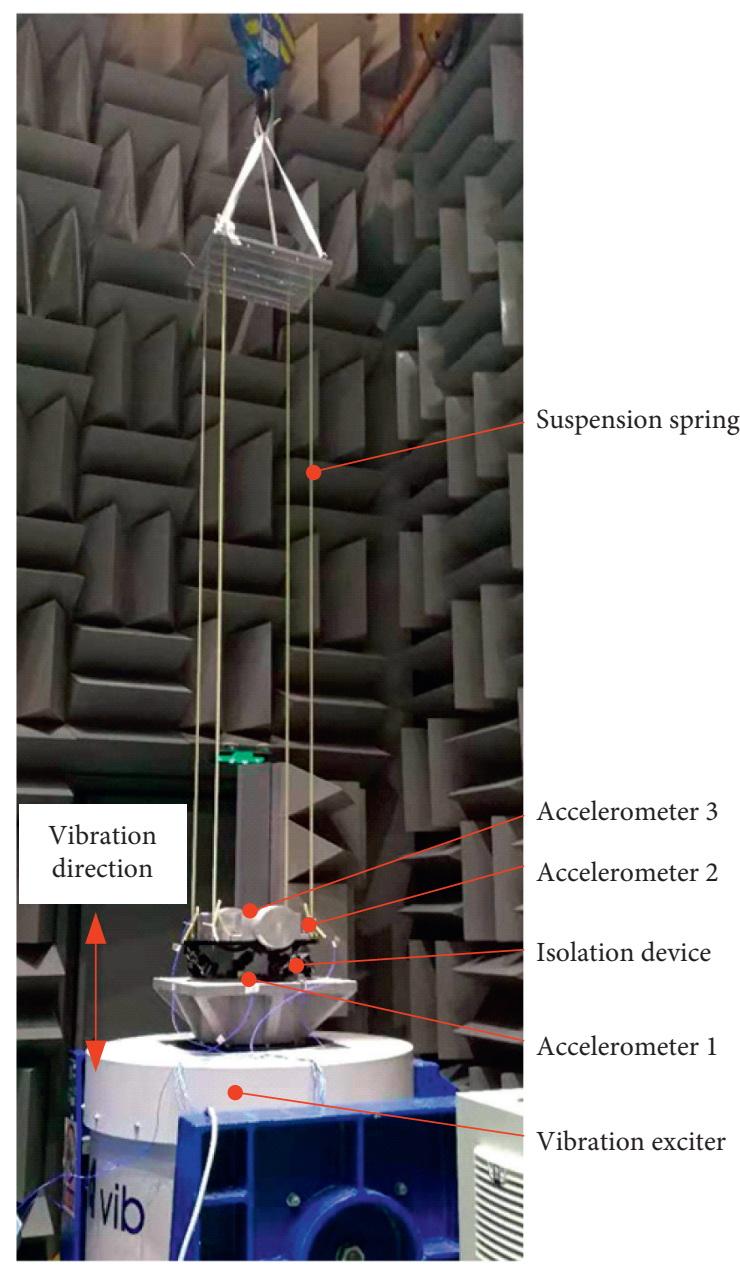

FIGURE 12: The picture of the experimental apparatus.

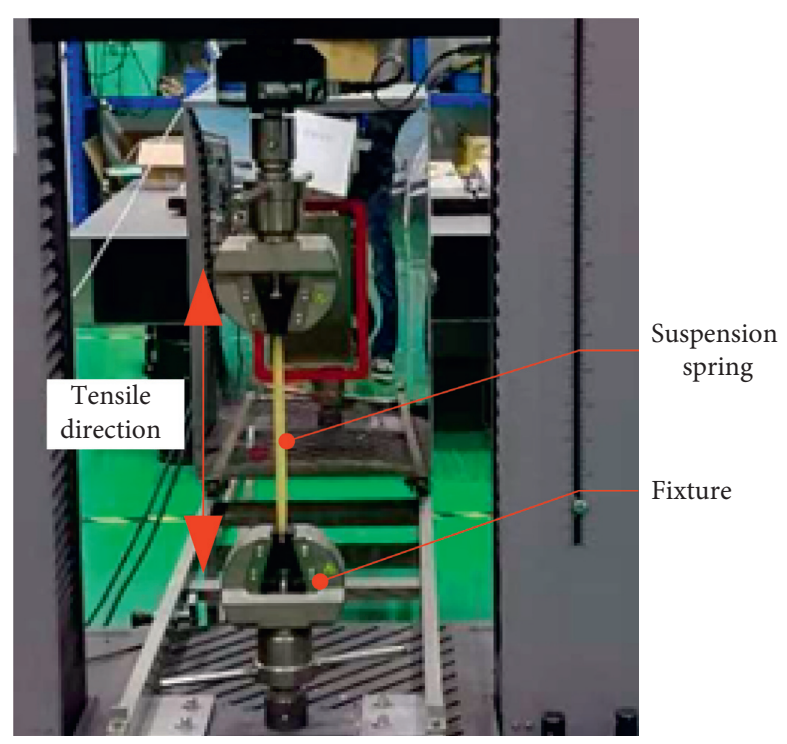

FIgURE 13: Picture of strip stiffness test experiment.

that there is no coupling in this direction. The amplification factor at the resonance frequency is less than 2 times.

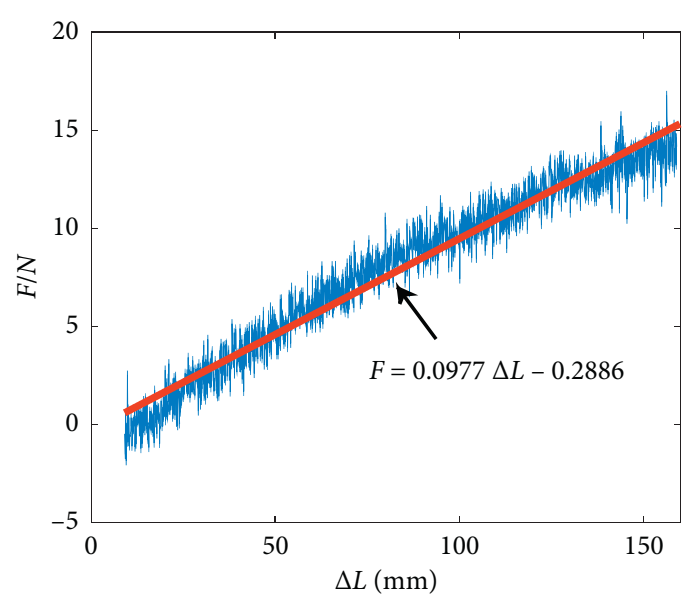

FIgURE 14: The force-deflection curve for a strip of the initial length of $410 \mathrm{~mm}$.

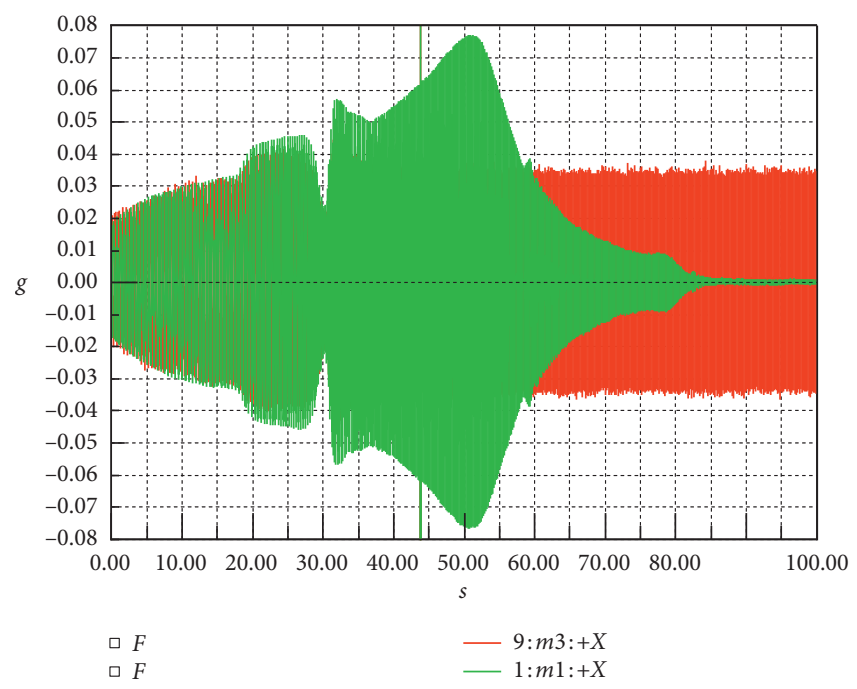

FIgURE 15: $X$-direction acceleration curve.

4.4. Frequency-Domain Results. The transmissibilities in the three directions are shown in Figures 18 to 20. Both the experimental and theoretical results are plotted. Overall, the proposed vibration isolation device has excellent vibration attenuation performance. More than $20 \mathrm{~dB}$ attenuation is obtained at the main frequency of CMG, which is $100 \mathrm{~Hz}$. There are slight deviations of the theoretical and experimental results in $X$-direction and $Y$-direction, though the resonance frequency is basically accurate. The reason for the deviation is possibly the coupling and the joint friction or assembly error. Nevertheless, the theoretical results can be used to predict such kind of vibration isolation device in engineering, since the trends are in good consistency and the deviations only occur at frequencies of small range.

In the $Z$-direction transmissibility, the theoretical and experimental results are in superb accordance. This is mainly because, as mentioned above, there is no coupling in this direction. In Figure 20, a transmissibility of a vibration isolation system with Kevin model is also plotted for comparison convenience. The curve is plotted by assuming a 


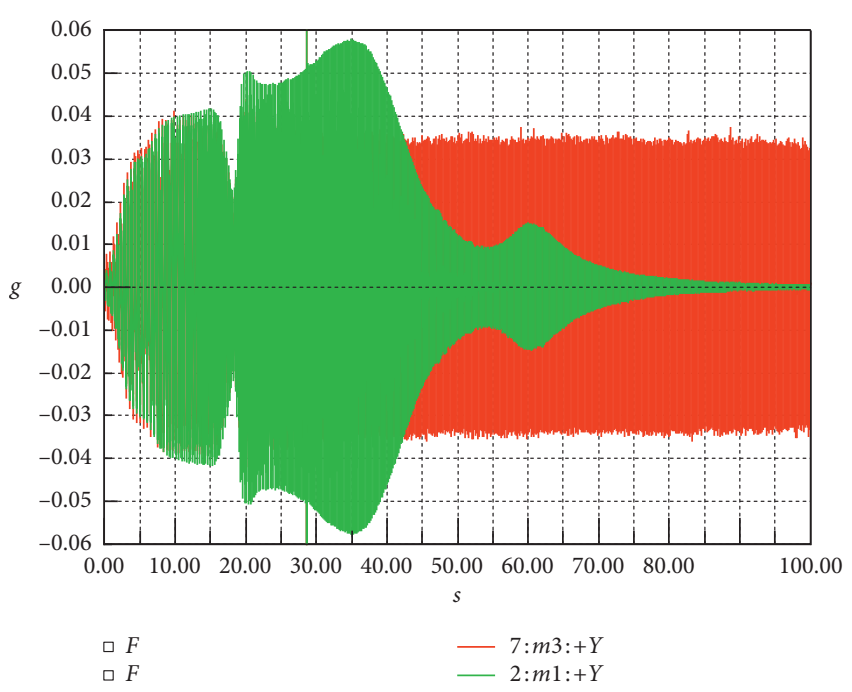

Figure 16: $Y$-direction acceleration curve.

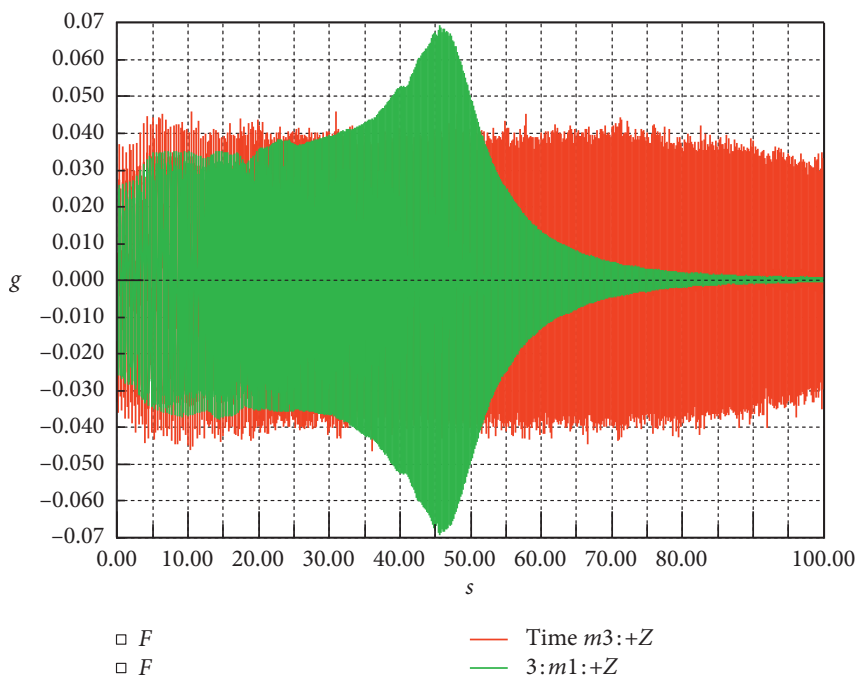

FIgURE 17: Z-direction acceleration curve.

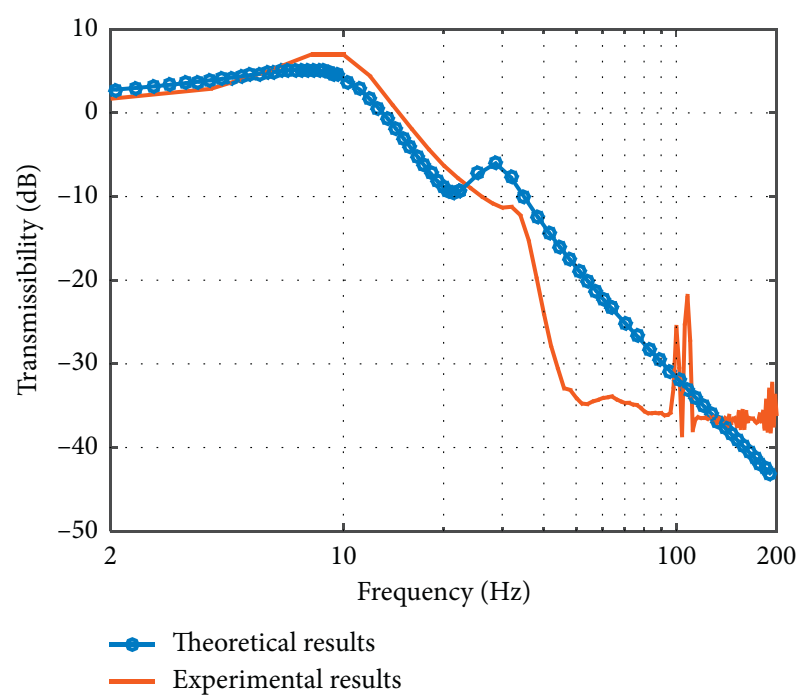

Figure 18: $X$-direction vibration isolation system transmission rate.

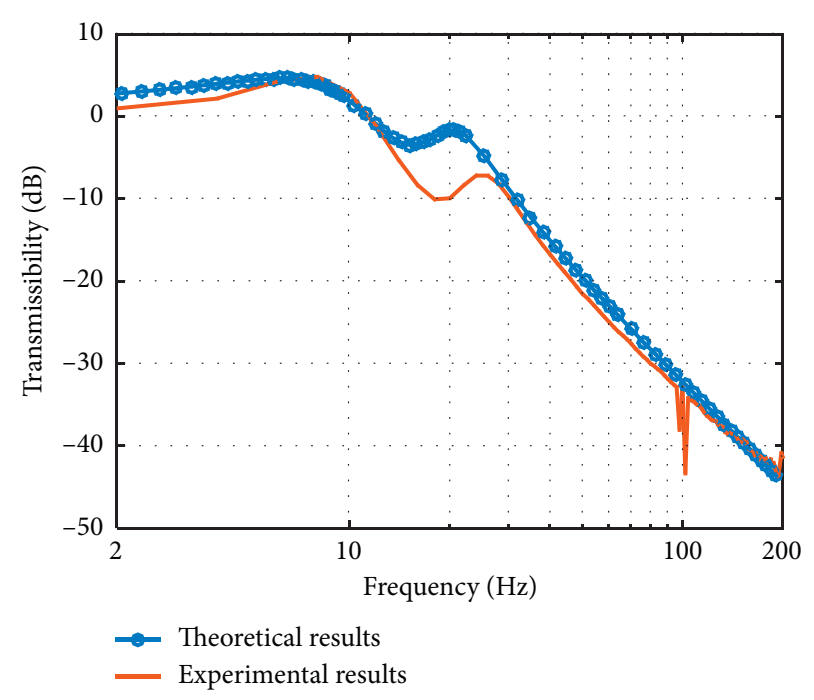

Figure 19: $Y$-direction vibration isolation system transmission rate.

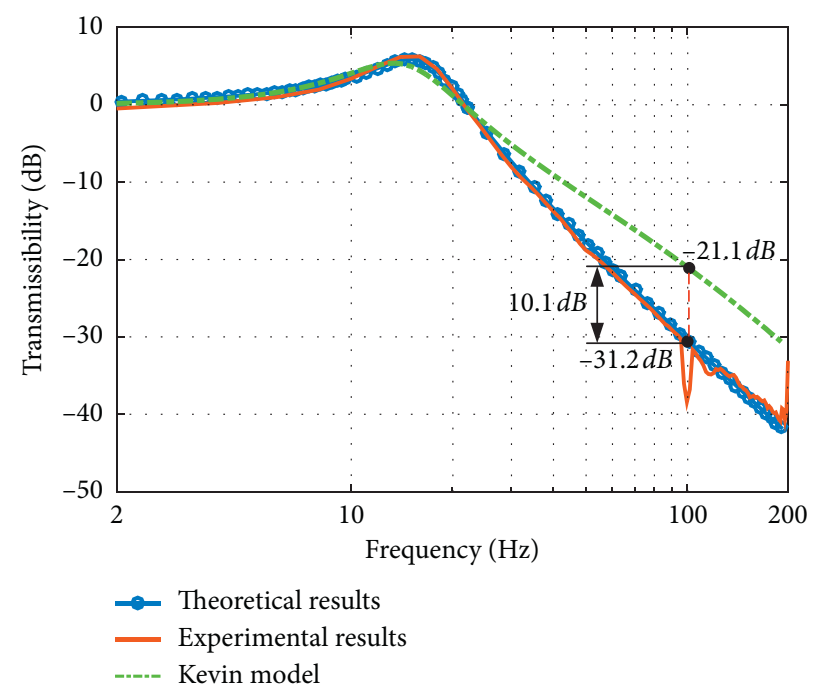

Figure 20: Comparison of the transmissibility of the proposed vibration isolation device with that of a Kevin model in $Z$-direction.

same resonance amplification factor for both systems. It can be seen from the figure that the device proposed in this paper can provide better vibration isolation performance at the main frequency of the CMG. The device with relaxation-type dampers may isolate $10 \mathrm{~dB}$ more vibration than that with a Kevin model according to the experimental results. Moreover, the amplification factor at resonance frequency of this device is below $10 \mathrm{~dB}$, which is an excellent index compared with traditional vibration isolation systems on market.

4.5. Effect of the Excitation Amplitude. In fact, the model studied in this paper is a linear system. In other words, the amplitude of excitation is irrelevant to the vibration isolation performance. However, this feature is verified experimentally for the sake of completeness. The excitation amplitude 


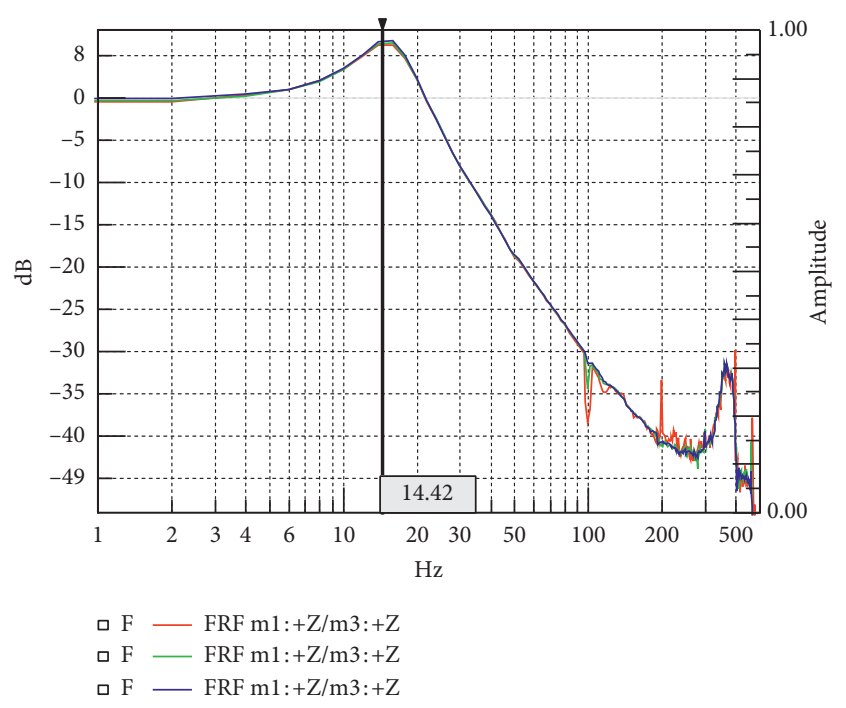

Figure 21: Acceleration transfer rate along $Z$-direction in different excitation amplitudes.

is varied as $10 \mathrm{mg}, 20 \mathrm{mg}$, and $30 \mathrm{mg}$ in $Z$-direction and the transmissibilities are plotted in Figure 21 for different excitation amplitudes. From the figure, one can easily distinguish that the amplitude of the excitation has rare effect on the vibration isolation performance.

\section{Conclusions}

A microvibration suppression device is proposed based on a relaxation-type damper and cubic configured Stewart platform in this paper. Both theoretical and experimental investigations are carried out. Some conclusions can be drawn as follows:

(1) The height between the mass center of the CMG and the upper platform of the device is the main factor that induces coupling effect; this parameter should be chosen carefully when designing this kind of device.

(2) The proposed device can provide more than $30 \mathrm{~dB}$ vibration attenuation at the main frequency of the CMG and less than $10 \mathrm{~dB}$ amplification at resonance, which is better than that of a traditional linear one.

(3) The excitation amplitude has rare effect on the vibration isolation performance and the device can function in a wide amplitude range.

\section{Data Availability}

The data are available upon request to the corresponding author.

\section{Conflicts of Interest}

The authors declare that they have no conflicts of interest.

\section{Acknowledgments}

The authors acknowledge the financial support from the National Natural Science Foundation of China under Grant no. 51875363 .

\section{References}

[1] H. P. Frisch, "Thermally induced vibrations of long thinwalled cylinders of open section," Journal of Spacecraft and Rockets, vol. 7, no. 8, p. 897, 1970.

[2] Z. Shen, Q. Tian, X. Liu, and G. Hu, "Thermally induced vibrations of flexible beams using Absolute Nodal Coordinate Formulation," Aerospace Science and Technology, vol. 29, no. 1, pp. 386-393, 2013.

[3] B. Clapp, J. Sills, and C. Voorhees, "Hubble space telescope pointing performance due to micro-dynamic disturbances from the NICMOS cryogenic cooler," AIAA J, 2002.

[4] N. Jedrich, D. Zimbelman, M. Turczyn, et al., "Cryo cooler induced micro-vibration disturbances to the hubble space telescope," 2002.

[5] H.-U. Oh, K.-J. Lee, and M.-S. Jo, "A passive launch and onorbit vibration isolation system for the spaceborne cryocooler," Aerospace Science and Technology, vol. 28, no. 1, pp. 324-331, 2013.

[6] J. Firth and J. Black, "Vibration interaction in a multiple flywheel system," Journal of Sound and Vibration, vol. 331, no. 7, pp. 1701-1714, 2012.

[7] H.-U. Oh, S. Taniwaki, N. Kinjyo, and K. Izawa, "Flywheel vibration isolation test using a variable-damping isolator," Smart Materials and Structures, vol. 15, no. 2, p. 365, 2006.

[8] C. Gurrisi, R. Seidel, S. Dickerson, S. Didziulis, P. Frantz, and K. Ferguson, "Space Station Control Moment Gyroscope Lessons Learned," in Proceedings of the 40th Aerospace Mechanisms Symposium, NASA Kennedy Space Center, Cocoa Beach, FL, USA, May 2010.

[9] D. Yu, G. Wang, and Y. Zhao, "On-orbit measurement and analysis of the micro-vibration in a remote-sensing satellite," Advances in Astronautics Science and Technology, vol. 1, no. 2, pp. 191-195, 2018.

[10] A. J. Bronowicki, "Vibration isolator for large space telescopes," Journal of Spacecraft and Rockets, vol. 43, no. 1, pp. 45-53, 2006.

[11] L. P. Davis, J. F. Wilson, R. E. Jewell, J. J. Roden, "Hubble space telescope reaction wheel assembly vibration isolation system," NASA report, 1986.

[12] K. J. Pendergast and C. J. Schauwecker, "Use of a passive reaction wheel jitter isolation system to meet the advanced $\mathrm{X}$-ray astrophysics facility imaging performance requirements," Proceedings of SPIE - The International Society for Optical Engineering, vol. 3356, 1998.

[13] L. Li, L. Tan, L. Kong, D. Wang, and H. Yang, "The influence of flywheel micro vibration on space camera and vibration suppression," Mechanical Systems and Signal Processing, vol. 100 , pp. 360-370, 2018.

[14] R. G. Cobb, J. M. Sullivan, A. Das et al., "Vibration isolation and suppression system for precision payloads in space," Smart Materials and Structures, vol. 8, no. 6, p. 798, 1999.

[15] J. Spanos, Z. Rahman, and G. Blackwood, "A Soft 6-axis active vibration isolator," in Proceedings of the 1995 American Control Conference - ACC'95, Seattle, WA, USA, June 1995.

[16] Y. Zhang, M. Li, and J. Zhang, "Vibration control for rapid attitude stabilization of spacecraft," IEEE Transactions on Aerospace and Electronic Systems, vol. 53, no. 3, p. 1308, 2017.

[17] Y. Zhang, Y. Zang, M. Li, Y. Wang, and W. Li, "Active-passive integrated vibration control for control moment gyros and its application to satellites," Journal of Sound and Vibration, vol. 394, p. 1, 2017.

[18] M. Li, Y. Zhang, Y. Wang, Q. Hu, and R. Qi, “The pointing and vibration isolation integrated control method for optical 
payload," Journal of Sound and Vibration, vol. 438, p. 441, 2019.

[19] J. Zhang, Z. Guo, Y. Zhang, L. Tang, and X. Guan, "Inner structural vibration isolation method for a single control moment gyroscope," Journal of Sound and Vibration, vol. 361, pp. 78-98, 2016.

[20] B. J. Kawak, "Development of a low-cost, low micro-vibration CMG for small agile satellite applications," Acta Astronautica, vol. 131, p. 113, 2017.

[21] Z.-Q. Lu, D. Wu, H. Ding, and L.-Q. Chen, "Vibration isolation and energy harvesting integrated in a Stewart platform with high static and low dynamic stiffness," Applied Mathematical Modelling, vol. 89, pp. 249-267, 2021. 